'Departamento de Psicología, Universidad de La Frontera. Temuco, Chile.

${ }^{2}$ Facultad de Ciencias Agropecuarias y Forestales, Universidad de La Frontera. Temuco, Chile.

${ }^{3}$ Centro de Excelencia en Psicología Económica, Universidad de La Frontera. Temuco, Chile.

${ }^{4}$ Núcleo Científico y Tecnológico en Biorecursos (BIOREN-UFRO), Universidad de La Frontera. Temuco, Chile.

${ }^{5}$ Núcleo Científico y Tecnológico de Ciencias Sociales, Universidad

de La Frontera. Temuco, Chile. aPsicóloga. Candidata a Doctora en Psicología.

bPsicóloga. Doctora en Psicología. Ingeniera agrónoma. Doctora en Ciencias Empresariales.

Este estudio recibió el apoyo del CEPEC (Centro de Excelencia

en Psicología Económica y del Consumo de la Universidad de la Frontera), de CONICYT PFCHA Doctorado Nacional 2015-

21150294 y CONICYT PFCHA Doctorado Nacional 201521150294.

Las autoras declaran no tener conflictos de interés.

Recibido el 26 de marzo de 2019, aceptado el 22 de enero de 2020.

Correspondencia a: Regina M. Navarro Departamento de Psicología, Universidad de La Frontera. Casilla 54D, Temuco 4780000, Chile.

regina.navarro@ufrontera.cl.

\section{Autopercepción de salud en adultos mayores: moderación por género de la situación financiera, el apoyo social de amigos y la edad}

\author{
REGINA M. NAVARRO ${ }^{1,3, a}$, CAMILA SALAZAR-FERNÁNDEZ ${ }^{1, \mathrm{~b}}$, \\ BERTA SCHNETTLER ${ }^{2,3,4, \mathrm{c}}$, MARIANELA DENEGRI ${ }^{1,3,5, \mathrm{~b}}$
}

\section{The influence of financial situation and friends' support on health self-perception of older adults}

Background: The Chilean population is aging rapidly. This socio-demographic change imposes psychosocial and economic challenges that are relevant for the health care of older people. Aim: To assess the association between perceived financial situation, perceived social support by friends, and age with health self-perception in older people. Material and Methods: A questionnaire about health self-perception, financial situation, and social support perceived by friends was applied to 383 occupationally active adults aged $68 \pm 5$ years (66\% women) living in urban areas of Southern Chile. A gender-based multi-group path model was specified, which evaluated the association of health self-perception with the assessment of the financial situation, social support, and age. Results: The final model was moderated by gender. However, age was not associated with health self-perception. For men and women, the perceived financial situation showed a positive association with health self-perception. The perceived social support of friends was only significant for women. Conclusions: The financial situation and friends' support (among women) are factors that should be considered in the evaluation of the health perception of older adults.

(Rev Med Chile 2020; 148: 196-203)

Key words: Aged; Financial Management; Friends; Social Support.
Autopercepción de salud en adultos mayores: Moderación por género de la situación financiera, el apoyo social y la edad

$\tau$ a población latinoamericana está envejeciendo a un ritmo acelerado respecto a los países de Europa ${ }^{1,2}$. Esto se asocia a la disminución de la fecundidad, a la caída de las tasas de mortalidad, al incremento de la esperanza de vida y a los adelantos médicos y tecnológicos ${ }^{3}$. Este cambio sociodemográfico impone diversos desa- fíos psicosociales y económicos en Latinoamérica, específicamente, al considerar la baja cobertura de seguridad social y los altos niveles de pobreza que presentan los adultos mayores ${ }^{4,5}$. De esta manera, muchos adultos mayores deben seguir trabajando hasta edades avanzadas, debido a las bajas pensiones y escasos recursos económicos para solventar sus gastos ${ }^{6,7}$. En Chile, 16,5\% de la población es adulta mayor ${ }^{8}$ y en $30 \%$ de los hogares, ellos son los principales sostenedores ${ }^{9}$ y quienes, además, presentan altos niveles de endeudamiento y mo- 
rosidad a nivel nacional ${ }^{10}$. En el país, la Región de La Araucanía es la más envejecida respecto a su población, con $12,2 \%$ de mayores de 65 años $^{8} \mathrm{y}$, además presenta el mayor porcentaje de pobreza $(17,2 \%)$ a nivel nacional ${ }^{11}$.

Ante este panorama de vulnerabilidad psicosocial y económica al que se enfrentan los adultos mayores en Chile y Latinoamérica, urge garantizar la seguridad social, priorizando en el cuidado, prevención y promoción de su estado de salud ${ }^{6}$. Así, no bastaría con asegurar vidas más largas, sino que estas deben ser también más saludables. Con relación a esto, mayor atención se debe prestar a los factores que contribuyen y potencian la mantención de la autonomía y la funcionalidad ${ }^{3}$, las cuales corresponden a las metas del desarrollo evolutivo en la adultez mayor ${ }^{12}$.

En atención al logro de estas metas, la Organización Mundial de la Salud (OMS) reconoce como prioritaria la promoción de un envejecimiento activo y lo define como "un proceso de optimización de las oportunidades de salud, participación y seguridad con el fin de mejorar la calidad de vida de las personas a medida que envejecen" ${ }^{13}$. El envejecimiento activo considera las limitaciones propias del envejecimiento biológico, buscando un buen estado de salud física y mental, la mantención de las capacidades cognitivas, el control personal, la autonomía y las competencias financieras y sociales $^{3,14,15}$. A partir del alcance de las metas del envejecimiento activo, se espera que los adultos mayores autoevalúen más positivamente su estado de salud ${ }^{4}$.

El estado de salud es la principal preocupación de las personas a medida que van envejeciendo ${ }^{16,17}$ y la autovaloración de la misma tiene un efecto importante en el estado de salud y bienestar general ${ }^{18}$. Se ha demostrado que en la medición de la percepción del estado de salud convergen elementos tanto psicosociales, económicos, como de salud ${ }^{16,19,20}$, siendo esta evaluación una estimación confiable de la valoración global de cada individuo $^{19,21}$. En la valoración del estado de salud general, físico, mental ${ }^{17,22}$ y de la salud subjetiva o autopercepción de salud ${ }^{2,22,23}$, se ha encontrado que variables como la situación financiera percibida $^{24}$, el apoyo social percibido ${ }^{16}$, la edad ${ }^{25} \mathrm{y}$ el género ${ }^{26}$ juegan un rol esencial. Sin embargo, poco se sabe de la influencia conjunta de estas variables en adultos mayores y menos en contextos latinoamericanos.

\section{Variables que se asocian a la autopercepción de salud en la adultez mayor}

Además del estado de salud, otra de las principales preocupaciones de los adultos mayores es su situación financiera ${ }^{6,24,27}$. Concretamente, si no tienen el dinero suficiente, no podrán solventar los gastos para la compra de medicamentos o tratamientos de sus condiciones de salud más frecuentes y asociadas al envejecimiento biológico (ej.: hipertensión arterial) ${ }^{28}$. Si, además, se consideran las brechas económicas que existen entre hombres y mujeres en Latinoamérica, esta situación se agudiza para mujeres ${ }^{29}$. Un estudio reciente encontró que la insatisfacción con las finanzas mostraba mayor asociación con la autopercepción de salud en mujeres que en hombres, aumentando la fuerza de esta relación con la edad ${ }^{30}$.

Otra variable que ha demostrado una asociación con la autopercepción del estado de salud en adultos mayores es el apoyo social que reciben de amigos ${ }^{16,23,31}$. Así, lo más relevante en esta etapa es la calidad más que la cantidad de relaciones de amistad $^{23}$. El apoyo social de amigos ha mostrado un comportamiento diferenciado de acuerdo al género ${ }^{32,33}$. Dado que las mujeres son capaces de establecer vínculos emocionales más profundos y duraderos, esto actuaría potenciando su autoconfianza y servirían como un factor protector frente a depresión y los sentimientos de dependencia psicológica e inutilidad, los cuales están asociados a una autopercepción de salud negativa ${ }^{34}$.

Finalmente, con el aumento de la edad también aumentan los problemas de salud, la sobrecarga funcional, hay mayor vulnerabilidad, aumentan los eventos estresantes y, por tanto, la autopercepción de salud es más negativa ${ }^{35,36}$. Estas dificultades asociadas al aumento de la edad han mostrado diferencias de acuerdo al género ${ }^{26,30}$. Así, las mujeres de mayor edad tienden a evaluar más negativamente su estado de salud que los hombres.

Considerando los antecedentes planteados, el objetivo de este estudio es estimar la relación entre la situación financiera percibida, el apoyo social percibido de amigos y la edad con la autopercepción de salud en adultos mayores activos, diferenciando estas relaciones entre hombres y mujeres. 


\section{Método}

\section{Diseño y participantes}

Bajo un diseño transversal-correlacional, se invitó a participar a adultos mayores con envejecimiento activo (ej.: participación laboral y en clubes) entre 60 y 76 años en la Región de La Araucanía (Chile), todos de procedencia urbana. Se optó por muestreo por conveniencia, debido a la desconfianza que presentan los adultos mayores frente a la solicitud de datos personales, pues temen ser manipulados financiera y políticamente. El contacto con los adultos mayores fue facilitado por líderes de la mesa de adultos mayores de La Araucanía. La participación fue voluntaria y no hubo incentivos.

\section{Instrumentos}

\section{Situación financiera percibida}

Se define la situación financiera percibida como la apreciación de la capacidad personal para poder hacer frente a las deudas que se tienen, o de la liquidez disponible para pagarlas ${ }^{22}$. Se evaluó con el ítem: ¿Cómo juzgaría la situación financiera actual de su familia?, las alternativas de respuesta fluctúan entre 1 y 5 (Muy difícil, Difícil, Regular, Buena y Muy buena). Los mayores puntajes reflejan una mejor autoevaluación de la situación financiera actual.

\section{Apoyo social percibido de amigos}

El apoyo social percibido de amigos se refiere al apoyo emocional disponible por parte de amigos $^{33}$. Se empleó el promedio de la subescala de Zimet (1988), validada en Chile ${ }^{39}$ y que consta de 4 ítems (Tabla 1). Sus opciones de respuesta oscilan entre 1 y 5 (Totalmente en desacuerdo a Totalmente de acuerdo), así, mayores puntajes indican mayor percepción de apoyo social recibido de amigos.

\section{Autopercepción del estado de salud}

Se define como la percepción personal o subjetiva del estado global de salud, involucrando el estado de bienestar físico, mental y social ${ }^{19,35}$. Fue evaluada con el ítem: ¿Cómo juzgaría su estado de salud actual?, usado en estudios en Brasil $^{19} \mathrm{y}$ México $^{35}$. Este ítem fue evaluado con respuestas que van desde 1 a 5 (Muy difícil, Difícil, Regular, Buena y Muy buena). Mayores puntajes reflejaron una mejor autopercepción de salud.

Tabla 1. Estadísticos descriptivos de las variables de interés y diferencia de medias

\begin{tabular}{|c|c|c|c|c|c|}
\hline \multirow[b]{2}{*}{ Variables } & \multicolumn{2}{|c|}{$\begin{array}{l}\text { Mujeres } \\
(n=258)\end{array}$} & \multicolumn{2}{|c|}{$\begin{array}{l}\text { Hombres } \\
(n=125)\end{array}$} & \multirow{2}{*}{$\begin{array}{r}\text { Diferencia } \\
\text { de género } \\
\text { Prueba t }\end{array}$} \\
\hline & Media & $\begin{array}{l}\text { Desviación } \\
\text { típica }\end{array}$ & Media & $\begin{array}{l}\text { Desviación } \\
\text { típica }\end{array}$ & \\
\hline $\begin{array}{l}\text { Situación financiera percibida: } \\
\text { ¿Cómo juzgaría la situación financiera actual de su } \\
\text { familia? }\end{array}$ & 2,555 & 0,987 & 2,895 & 1,010 & $-3,094 * *$ \\
\hline Apoyo social percibido de amigos (promedio): & 3,556 & 1,345 & 3,266 & 1,391 & $1,928^{*}$ \\
\hline $\begin{array}{l}\text { 1. ¿Cómo juzgaría la situación financiera actual de su } \\
\text { familia? }\end{array}$ & 3,686 & 1,307 & 3,145 & 3,145 & $3,681^{* *}$ \\
\hline $\begin{array}{l}\text { 2. Tengo la seguridad de que mis amigos(as) tratan de } \\
\text { ayudarme }\end{array}$ & 3,700 & 1,340 & 3,282 & 1,411 & $2,803^{* *}$ \\
\hline $\begin{array}{l}\text { 3. Puedo contar con mis amigos(as) cuando tengo } \\
\text { problemas }\end{array}$ & 3,556 & 1,345 & 3,266 & 1,391 & $1,951^{*}$ \\
\hline 4. Puedo conversar de mis problemas con mis amigos(as) & 3,390 & 1,395 & 3,040 & 1,450 & $2,264^{*}$ \\
\hline $\begin{array}{l}\text { Autopercepción de salud: } \\
\text { ¿Cómo juzgaría su estado de salud actual? }\end{array}$ & 2,763 & 1,009 & 3,024 & 1,077 & $-2,251 *$ \\
\hline Edad & 68,062 & 4,548 & 69,072 & 4,704 & $-1,991^{*}$ \\
\hline
\end{tabular}

Nota: ${ }^{* *} \leq 0,001 ; * \leq 0,005$. 


\section{Cuestionario sociodemográfico}

Se consultó a cada participante por su género, edad, ocupación, estado civil y nivel educacional.

\section{Procedimiento}

Los participantes fueron contactados en clubes de adultos mayores, en sus residencias o lugares de trabajo, mediante llamado telefónico previo. Habiendo informado de la voluntariedad de la participación y firmado el consentimiento informado, los encuestadores procedieron con la recolección de datos. El procedimiento fue aprobado por el Comité Ético Científico de la Universidad de La Frontera.

\section{Análisis de datos}

Con el programa Stata 15.1 se revisaron los estadísticos descriptivos de las variables de interés y mediante una prueba-t de muestras independientes se indagaron diferencias por género. Luego, se especificaron modelos de sendero que daban cuenta de la relación entre la satisfacción financiera percibida, el apoyo de amigos, el género y la edad con la autopercepción de salud. Estos análisis fueron ajustados como un modelo de senderos multigrupo, utilizando el género como moderador, así se obtuvo un modelo para hombres y mujeres. Se utilizaron análisis de senderos debido a que permiten la estimación de la magnitud y la significación de las asociaciones hipotetizadas cuando se cuenta con medidas de un solo ítem o con el promedio de una escala. Estas medidas de un solo ítem, más sencillas, permitieron garantizar la comprensión de los participantes, dado que cerca de $66 \%$ de los adultos mayores en Chile no ha completado el nivel de enseñanza obligatoria ${ }^{40}$. Si bien el objetivo de esta investigación es evaluar la relación entre la situación financiera percibida, el apoyo social percibido de amigos y la edad con la autopercepción de salud, con los modelos de senderos es posible, también, examinar configuraciones alternativas, por ejemplo, si alguna variable no correlaciona puede restringirse ese parámetro.

Los modelos fueron evaluados de acuerdo a los criterios convencionales de ajuste global: $\mathrm{CFI}$ y TLI $>0,95$ y RMSEA $<0,08^{41}$ y se utilizó el test de Wald para evaluar las diferencias estadísticas entre hombres y mujeres.

\section{Resultados}

\section{Análisis preliminares}

El 86\% de los participantes provenían de Temuco y $14 \%$ de Angol. El promedio de edad fue de 68,39 años (DE: 4,61 y $65,8 \%$ eran mujeres. Los participantes se encontraban distribuidos en los niveles socioconómicos bajo (16,67\%), medio bajo $(20,22 \%)$, medio $(43,44 \%)$ y medio alto $(19,67)$, de acuerdo a los criterios de la Sociedad europea de opinión e investigación de mercados (ESOMAR). El análisis de confiabilidad de la escala de apoyo social percibido de amigos, obtenido con el alfa de Cronbach fue muy buena $(0,919)$.

\section{Análisis descriptivos}

A nivel descriptivo se encontró que los hombres presentaron significativamente mayor edad, satisfacción financiera y una mejor autopercepción del estado de salud. Las mujeres presentaron mayor apoyo social de amigos (Tabla 1).

\section{Modelo de sendero moderado por género}

El modelo inicial encontró que, tanto para hombres como para mujeres, la edad no estaba relacionada significativamente con la autopercepción de salud. Debido a esto, se procedió a estimar un modelo más sencillo que restringiera la relación de la edad con la autopercepción de salud a cero. Esta restricción permitió la evaluación del ajuste del modelo al liberar un grado de libertad.

Este nuevo modelo multigrupo presentó muy buenos indicadores de ajuste a los datos: $\chi^{2}(2)=$ 2,267; $p=0,322$; CFI $=0,997$; TLI $=0,991$; RM$\mathrm{SEA}=0,027 ; \mathrm{SRMR}=0,022$, lo cual, confirmó la moderación del género (Figura 1). Los resultados indicaron que, para hombres y mujeres, la situación financiera percibida se relacionó positiva y significativamente con la autopercepción de salud en ambos géneros (Tabla 2 y Figura 1). Más importante, el apoyo social percibido de amigos solo fue significativo para mujeres, encontrándose una asociación positiva. Estas diferencias entre hombres y mujeres no fueron estadísticamente significativas de acuerdo al test de Wald, $\chi^{2}(1)=$ $0,927, \mathrm{p}>0,05$. Ambos modelos explicaron más del $20 \%$ de la varianza. 
Tabla 2. Asociaciones entre situación financiera, apoyo social de amigos y edad con autopercepción del estado de salud moderado por género

\begin{tabular}{|lcccc|}
\hline & \multicolumn{4}{c}{$\begin{array}{c}\text { Autopercepción del estado de salud } \\
\text { Hujombres }\end{array}$} \\
\hline Edad & B & IC 95\% & B & IC 95\% \\
\hline Situación financiera percibida & 0,010 & {$[-0,100 ; 0,120]$} & $-0,131$ & {$[-0,284 ; 0,021]$} \\
\hline Apoyo social percibido de amigos & $0,406^{* *}$ & {$[0,308 ; 0,505]$} & $0,445^{* *}$ & {$[0,300 ; 0,589]$} \\
$R^{2}$ & $0,134^{*}$ & {$[0,023 ; 0,244]$} & 0,036 & {$[-0,129 ; 0,202]$} \\
\hline
\end{tabular}

Nota: Significancia bilateral: ${ }^{*} \leq 0,005 ;{ }^{* *} \leq 0,001$.

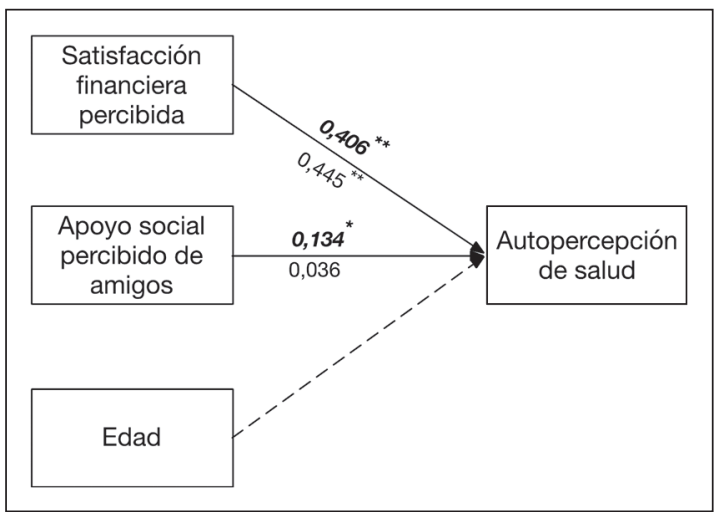

Figura 1. Satisfacción financiera percibida, apoyo social percibido de amigos y edad para adultos mayores hombres y mujeres. Los senderos para las participantes mujeres están representados en negrita y cursiva. ${ }^{* *} p<0,05$. La línea punteada representa el sendero fijado a 1,0 debido a su ausencia de significancia en el modelo previo.

\section{Discusión}

El objetivo de este estudio fue estimar la relación entre la situación financiera percibida, el apoyo social percibido de amigos y la edad con la autopercepción de salud en adultos mayores activos, diferenciando entre hombres y mujeres. Los resultados indicaron que, para hombres y mujeres, la situación financiera de los adultos mayores estaba asociada a una mejor autopercepción del estado de salud, mientras que el apoyo social de amigos solo mostró una relación significativa en mujeres. No se encontró una asociación entre la edad y la autopercepción del estado de salud.

La asociación entre la autopercepción de la situación financiera y el estado de salud en adultos mayores parece respaldar la importancia de contar con garantías económicas suficientes para la compra de medicamentos, la atención oportuna en servicios de salud y el seguimiento a dietas ${ }^{41}$, los cuales contribuyen al control de un buen estado de salud controlado. Ver mermada la capacidad financiera también impacta la salud mental, pues, de acuerdo con Lue, Chen y $\mathrm{Wu}^{42}$ y Krause ${ }^{43}$, la falta de recursos económicos podría ocasionar estrés financiero, ansiedad o depresión, situación que se agravaría en caso de sobreendeudamiento $y$ morosidad ${ }^{10}$.

Por su parte, el apoyo social de amigos ha demostrado ser un factor protector de la salud y de la longevidad ${ }^{23,44}$. Los resultados hallados amplían los obtenidos por Giles, Glonek, Luszcz y Andrews ${ }^{45}$, al detectar diferencias en el rol de la amistad por género. Al respecto, algunas investigaciones ${ }^{33,46}$ han planteado que, para las mujeres adultas mayores, las relaciones de amistad promueven la confianza y guían la formación de vínculos emocionales fuertes, los cuales proporcionan el apoyo para enfrentar los desafíos cotidianos. Además, las mujeres están más dispuestas a establecer nuevas relaciones de amistad, las cuales fortalecen su autoestima y permiten estrechar lazos de solidaridad entre ellas ${ }^{33,46}$. Estos hallazgos refuerzan la importancia de seguir estimulando la socialización entre los adultos mayores, dadas sus asociaciones positivas en la autopercepción del estado de salud.

La edad no se encontró asociada con la autopercepción de salud, tanto para hombres como para mujeres. Este resultado podría explicarse porque las asociaciones positivas entre la edad y la autopercepción de salud se han encontrado 
en estudios con adultos mayores sobre 80 años $^{23}$ y se atribuyen al rápido deterioro físico y cognitivo ${ }^{19,20,45}$. Así, podría no ocurrir lo mismo en adultos mayores de menor edad, como los del presente estudio.

Respecto a las fortalezas de este estudio se reconoce la importancia que tienen los factores económicos y psicosociales en la percepción de salud, diferenciando entre hombres y mujeres. Esto, fundamentalmente, debido a la desigual situación latinoamericana asociada al género del adulto mayor ${ }^{5}$. Además, la obtención de un buen tamaño muestral permite extrapolar estos hallazgos a otras regiones. Los resultados encontrados reflejan la realidad actual de los adultos mayores en la región más pobre de Chile, los cuales pueden orientar la implementación de políticas en salud pública (e.j.: apoyos instrumentales solidarios, estimulación de la integración social).

A pesar de sus fortalezas, esta investigación presenta algunas limitaciones, pues, al tratarse de un estudio transversal, no permite establecer causalidad. Debido a la alta desconfianza de los participantes, se decidió un muestreo no probabilístico de la población de adultos mayores de Chile, por lo cual, los resultados deben tomarse con cautela, ya que representan las características específicas de la muestra. Adicionalmente, las diferencias no significativas entre hombres y mujeres respecto al apoyo social percibido de amigos, parecen reflejar la desigualdad en la conformación muestral de ambos grupos. Otra limitación es el uso de ítems únicos para evaluar algunas de las variables de interés, con el fin de garantizar la comprensión y atención de los participantes. $\mathrm{Al}$ respecto, recomendamos que futuros estudios utilicen escalas confiables y validadas con adultos mayores. Estos estudios también deberán revisar el impacto de las relaciones de amistad en las redes sociales virtuales entre adultos mayores ${ }^{47}$, quienes cada vez más incursionan en estos medios.

La evidencia obtenida es un punto de partida oportuno para el desarrollo de investigaciones longitudinales utilizando muestras de mayor tamaño, las cuales permitirán obtener un panorama orientado a la prevención, promoción y atención de la salud de adultos mayores. Así también, estos hallazgos son relevantes para ser considerados por los equipos de salud, quienes deben contemplar los distintos aspectos de la realidad psicosocial y económica de las personas mayores, con el objetivo de promover un envejecimiento activo de la población.

Agradecimientos: Las autoras agradecen y hacen especial reconocimiento a la mesa del adulto mayor de la Región de La Araucanía y a la Sra. Matilde Jerez de Riquelme, su presidenta, por facilitar el contacto con diferentes agrupaciones de adultos mayores en las comunas de Angol y Temuco, así como a cada uno de los participantes en la presente investigación.

\section{Referencias}

1. Suárez R, Pescetto C. Sistemas de protección social para el adulto mayor en América Latina y el Caribe. Rev Panam Salud Publ 2005; 17 (56): 420-8.

2. Puga D, Rosero-Bixby L, Glaser K, Castro T. Red social y salud del adulto mayor en perspectiva comparada: Costa Rica, España e Inglaterra. Población y Salud en Mesoamérica 2007; 5 (1): 1-22.

3. OMS. Informe Mundial sobre el Envejecimiento y la Salud 2015; (1): 1-196.

4. Aranco N, Stampini M, Ibarrarán P, Medellín N. Panorama de envejecimiento y dependencia en América Latina y el Caribe. Banco Interamericano de Desarrollo. 2018; 4-101.

5. Huenchuan S, Guzmán M. Seguridad Económica y Pobreza en la vejez: Tensiones, expresiones y desafíos para políticas públicas. Cepal 2006; Santiago (Fondo de Población de las Naciones Unidas): 1-23.

6. HelpAge. Informe sobre el Envejecimiento y el desarrollo: Pobreza, independencia y las personas mayores en el mundo. En: HelpAge Internacional 2016; 1-20.

7. OECD, BID, World Bank. Panorama de las Pensiones: América Latina y El Caribe 2015; 1-182.

8. INE (Instituto Nacional de Estadísticas). Resultados definitivos Censo 2017. Gobierno de Chile 2017; 1-41.

9. Adimark E. Adultos Mayores en Chile: Ojos puestos en el futuro. Adimark Chile 2015; 1-31.

10. Superintendencia de Bancos e Instituciones Financieras (SBIF). Informe de endeudamiento de los clientes bancarios. SBIF 2015;1-27.

11. Casen. Pobreza y distribución de ingresos contenidos. Ministerio de Desarrollo Social 2017. 1-84.

12. Baltes $P$, Staudinger U, Lindenberger U. Lifespan psychology: Theory and application to intellectual functioning. Annu Rev Psychol 1999; 50: 471-507.

13. Organización Mundial de la Salud (OMS). Enveje- 
cimiento activo: un marco político. Rev Esp Geriatr Gerontol 2002; 37 (52): 74-105.

14. Fernández-Ballesteros García R, Zamarrón Casinello MD, López Bravo MD, Molina Martínez MÁ, Díez Nicolás J, Montero López P, et al. Envejecimiento con éxito: criterios y predictores. Psicothema 2010; 22 (4): 641-7.

15. Baltes P, Baltes M. Successful Aging: Perspectives from the behavioral sciencies. Cambridge: Cambridge University Press. 1990.

16. Gallardo L, Barrón A, Sánchez E, Arias A. Dimensiones estructurales y funcionales del apoyo social y salud en personas mayores en Chile. Rev Mex Psicol 2014; 31 (2): 187-97.

17. Cuadra-Peralta A, Medina EF, Guerrero KJS. Relación de bienestar psicológico, apoyo social, estado de salud física y mental con calidad de vida en adultos mayores en la ciudad de Arica. Límite 2016; 11 (35): 56-67.

18. Mauricio Ocampo J. Self-rated health: Importance of use in elderly adults. Colombia Médica 2010; 41 (3): 275-89.

19. Pavao ALB, Werneck GL, Campos MR. Autoavaliação do estado de saúde e a associação com fatores sociodemográficos, hábitos de vida e morbidade na população: um inquérito nacional self-rated health and the association with social and demographic factors, health behavior, and morbidity. Cad Saúde Pública 2013; 29 (4): 723-34.

20. Bøen H, Dalgard OS, Bjertness E. The importance of social support in the associations between psychological distress and somatic health problems and socio-economic factors among older adults living at home: A cross sectional study. Geriatrics 2012; 12 (27): 1-12.

21. Damian J, Ruigomez A, Pastor V, Martin-Moreno JM. Determinants of self-assessed health among Spanish older people living at home. J Epidemiol Community Health 1999; (53): 412-6.

22. Rodríguez-Laso A, Abellan A, Sancho M, Pujol R, Montorio I, Díaz-Veiga P. Perceived economic situation, but not education level, is associated with disability prevalence in the Spanish elderly: Observational study. Geriatris 2014; 14 (1): 2-11.

23. Waldinger RJ, Schulz MS. What's love got to do with it? Social functioning, perceived health, and daily happiness in married octogenarians. Psychol Aging 2010; 25 (2): 422-31.

24. Arber S, Fenn K, Meadows R. Subjective financial well-being, income and health inequalities in mid and later life in Britain. Soc Sci Med 2014; 100: 12-20.

25. Deeks A, Lombard C, Michelmore J, Teede H. The effects of gender and age on health related behaviors.
Public Health 2009; 8 (213): 1-8.

26. Roy K, Chaudhuri A. Influence of socioeconomic status, wealth and financial empowerment on gender differences in health and healthcare utilization in later life: evidence from India. Soc Sci Med 2008; 66: 195162.

27. Scott SB, Jackson BR, Bergeman CS. What contributes to perceived stress in later life? A recursive partitioning approach. Psychology \& Aging 2011; 26 (4): 830-43.

28. Pérez V, Sierra F. Biología del envejecimiento. Rev Med Chile 2009; 137: 296-302.

29. Huenchuan S. Envejecimiento, solidaridad y protección social en América Latina y el Caribe. La hora de avanzar hacia la igualdad. CEPAL (Com. Naciones Unidas) 2013; 3-192.

30. Otaki N, Tanino N, Yokoro M, Yano M, Akita M, Uemura H, et al. Relationship between economic security and self-rated health in elderly Japanese residents living alone. J Nutr Health Ageing 2017; 22 (6): 1-5.

31. Fiori KL, Antonucci TC, Cortina KS. Social network typologies and mental health among older adults. J Gerontol 2006; 61 (1): 25-32.

32. Siu O, Phillips DR. A study of family support, frienshipand psychological wellbeing among older woman in Hong Kong. Int J Aging Hum Dev 2002; 55 (4): 299319.

33. Huechan S, Guzmán JM. Redes de apoyo social de las personas mayores: Marco conceptual. Repositorio Digital CEPAL. 2003: 35-70.

34. Adams RG, Blieszner R, Vries B. Definition of friendship in the third age: Age, gender, and study location effects. Journal of Aging Studies 2000; 14 (1): 117-33.

35. Angel RJ, Frisco M, Angel JL, Chiriboga DA, Angel RJ, Angel JL. Financial strain and health among elderly mexican-origin individuals. J Health Soc Behav 2003; 44 (4): 536-51.

36. Sousa AC, Dias RC, Maciel ÁC, Guerra RO. Frailty syndrome and associated factors in community-dwelling elderly in Northeast Brazil. Arch Gerontol Geriatr 2012; 54 (2): 95-100.

37. Adimark. El Nivel Socioeconómico ESOMAR 2000; 1-8.

38. Ortiz MS, Baeza MJ. Propiedades psicométricas de una escala para medir apoyo social percibido en pacientes chilenos con diabetes tipo 2. Universitas Psychologica. 2011; 10(1):189-96.

39. Arnold M, Herrera, F, Massad, C, Thumala, D. Quinta encuesta nacional de inclusión y exclusión social de las personas mayores en Chile: Opiniones de la población chilena respecto al envejecimiento poblacional. Ediciones Servicio Nacional del Adulto Mayor: Santiago, Chile. 2018. 
40. Albala C, García C, Lera L. SABE 2000: Salud, bienestar y envejecimiento en Santiago, Chile. Organización Panamericana de la Salud (OPS) 2000; 3-94.

41. Marsh HW, Hau K-T, Wen Z. In search of golden rules: Comment on hypothesis-testing approches to setting cutoff values for fit indexes and dangers in overgeneralizing $\mathrm{Hu}$ and Bentler's (1999) findings. Struct Equ Modeling 2004; 11 (3): 320-41.

42. Lue B, Chen L, Wu S. Health, financial stresses, and life satisfaction affecting late-life depression among older adults: A nationwide, longitudinal survey in Taiwan. Arch Gerontol Geriatr 2010; 50 (1): 34-8.

43. Krause N. Financial strain, received support, anticipated support, and depressive symptoms in the people's Repu- blic of China. Psychol Ageing 1998; 13 (1): 58-68.

44. Arias C, Polizzi L. La amistad en la vejez. Funciones de apoyo y atributos del vínculo. Universidad de Buenos Aires. Buenos Aires, Argentina, 2011.

45. Giles L, Glonek G, Luszcz M, Andrews G. Effect of social networks on 10-year survival in very old Australians: The Australian longitudinal study of aging. Epidemiology Community Health 2005; (59): 574-9.

46. Arias CJ. El apoyo social en la vejez: La familia, los amigos y la comunidad. Rev Kairos 2013; 16 (4): 313-29.

47. Llorente-Barroso C, Viñarás-Abad M, Sánchez-Valle M. Mayores e Internet: La red como fuente de oportunidades para un envejecimiento activo. Comunicar 2015; 23 (45): 29-36. 